\title{
Gorenstein Quivers
}

\section{Vladimir Kirichenko and Makar Plakhotnyk}

Faculty of Mechanics and Mathematics, Kyiv National Taras Shevchenko University, Kyiv, Ukraine

E-mail address: vkir@univ.kiev.ua

E-mail address: Makar_Plakhotnyk@ukr.net

\begin{abstract}
We introduce a notion of Gorenstein quiver associated with a Gorenstein matrix. We study properties of such quivers. In particular, we show that any such quiver is strongly connected and simply laced. We use Perron-Frobenius theory of non-negative matrices for characterization of isomorphic Gorenstein quivers.
\end{abstract}

\section{Introduction}

The notion of exponent and Gorenstein matrix has origin in ring theory. It is important in the study of Gorenstein rings considered by H.Bass in 1963 (see [1]). In particular, these concepts are relevant to the study of Gorenstein tiled orders [5, Ch.7].

In this paper we introduce a notion of Gorenstein quiver associated with a Gorenstein matrix and study the properties of these quivers. In Section 2 we give a short survey of main results on semiprime right Noetherian semiperfect and semidistributive rings which lead to a concept of a Gorenstein matrix and preliminary results on Gorenstein matrices. In Section 3 we recall classical theorems of Perron and Frobenius on non-negative matrices which play important role in our characterization of isomorphic Gorenstein quivers. Finally, Section 4 contains our main results. Here we define a Gorenstein quiver associated with every Gorenstein matrix. We show that any such quiver is strongly connected and simply laced. We also give a characterization of isomorphic Gorenstein quivers. At the end we give some examples of Gorenstein quivers. 


\section{Preliminaries}

2.1. SPSD-rings. For convenience of the reader we recall main result on semiprime right Noetherian semiperfect and semidistributive rings (see [4, Ch.14]). We write SPSD-ring for semiperfect and semidistributive ring (see $[4$, Ch.14]).

Definition 2.1. A ring is called semimaximal if it is a semiperfect semiprime right Noetherian ring such that for each local idempotent $e \in A$ the ring $e$ Ae is a discrete valuation ring (not necessary commutative).

The following is a decomposition theorem for semiprime right Noetherian SPSD-rings.

Theorem 2.1. The following conditions for a semiperfect right Noetherian $S P S D$-ring are equivalent:

(a) the ring $A$ is semidistributive;

(b) the ring $A$ is a direct product of a semisimple Artinian ring and a semimaximal ring;

Theorem 2.2. Each semimaximal ring is isomorphic to a finite direct product of prime rings of the following form:

$$
\left(\begin{array}{cccc}
\mathcal{O} & \pi^{\alpha_{12}} \mathcal{O} & \cdots & \pi^{\alpha_{1 n}} \mathcal{O} \\
\pi^{\alpha_{21}} \mathcal{O} & \mathcal{O} & \cdots & \pi^{\alpha_{2 n}} \mathcal{O} \\
\cdots & \cdots & \cdots & \cdots \\
\pi^{\alpha_{n 1}} \mathcal{O} & \pi^{\alpha_{n 2}} \mathcal{O} & \cdots & \mathcal{O}
\end{array}\right)
$$

where $n \geqslant 1, \mathcal{O}$ is a discrete valuation ring with a prime element $\pi$, and $\alpha_{i j}$ are integers such that $\alpha_{i j}+\alpha_{j k} \geqslant \alpha_{i k}$ for all $i, j, k\left(\alpha_{i i}=0\right.$ for any $\left.i\right)$.

Definition 2.2. A matrix $\mathcal{E}=\left(\alpha_{i j}\right)$ is called exponent matrix if $\mathcal{E}$ satisfies the following two conditions:

- $\alpha_{i i}=0$ for $i=1, \ldots, n$;

- $\alpha_{i j}+\alpha_{j k}=\alpha_{i k}$ for $i, j, k=1, \ldots, n$.

An exponent matrix $\mathcal{E}$ is called reduced exponent matrix if $\alpha_{i j}+\alpha_{j i}>0$ for $i \neq j$.

Denote by $M_{n}(B)$ a ring of all $(n \times n)$-matrices with elements from a ring $B$. Let $\mathcal{O}$ be a discrete valuation ring with prime element $\pi$ and $\mathcal{M}=\pi \mathcal{O}=\mathcal{O} \pi$ is the unique maximal ideal of $\mathcal{O}, D$ is the classical division ring of fractions of $\mathcal{O}$.

Denote by $A=\left\{\mathcal{O}, \mathcal{E}=\left(\alpha_{i j}\right)\right\}$ the following subring of $M_{n}(D)$ : 


$$
A=\left(\begin{array}{cccc}
\mathcal{O} & \pi^{\alpha_{12}} \mathcal{O} & \ldots & \pi^{\alpha_{1 n}} \mathcal{O} \\
\pi^{\alpha_{21}} \mathcal{O} & \mathcal{O} & \ldots & \pi^{\alpha_{2 n}} \mathcal{O} \\
\ldots & \ldots & \ldots & \ldots \\
\pi^{\alpha_{n 1}} \mathcal{O} & \pi^{\alpha_{n 2}} \mathcal{O} & \ldots & \mathcal{O}
\end{array}\right)
$$

A ring $A$ is a semiperfect and semidistributive prime Noetherian ring with nonzero Jacobson radical (tiled order), see [4, Ch.14].

Let $A$ be a semiperfect ring with the Jacobson radical $R$. A ring $A$ is called reduced if $A / R$ is a direct product of division rings. In particular, a tiled order $A=\left\{\mathcal{O}, \mathcal{E}=\left(\alpha_{i j}\right)\right\}$ is reduced if and only if its exponent matrix $\mathcal{E}=\left(\alpha_{i j}\right)$ is reduced.

2.2. Gorenstein tiled orders and Gorenstein matrices. In this section we collect necessary statements about Gorenstein matrices.

Let $\mathcal{E}=\left(\alpha_{i j}\right) \in M_{n}(\mathbb{Z})$, where $\mathbb{Z}$ is the ring of integers.

Definition 2.3. A reduced exponent matrix $\mathcal{E}$ is called Gorenstein matrix if there exists a permutation $\tau$ of the set $\{1, \ldots, n\}$ such that $\alpha_{i j}+\alpha_{j \tau(i)}=$ $\alpha_{i \tau(i)}$ for all $i$ and $j$.

Theorem 2.3. [5, Ch.7] The following properties for reduced tiled order $A=\left\{\mathcal{O}, \mathcal{E}=\left(\alpha_{i j}\right)\right\}$ are equivalent:

(a) inj.dim $A_{A}=1$;

(b) $i n j . \operatorname{dim}_{A} A=1$;

(c) the exponent matrix $\mathcal{E}=\left(\alpha_{i j}\right)$ is Gorenstein.

Recall that a commutative ring is called Gorenstein if its injective dimension is finite.

If a reduced tiled order $A=\left\{\mathcal{O}, \mathcal{E}=\left(\alpha_{i j}\right)\right\}$ satisfies the conditions of Theorem 2.3 then it will be called Gorenstein tiled order. In particular, from $[5$, Ch.7] we obtain the following statement.

Corollary 2.1. If $A$ is a reduced Gorenstein tiled order then all rings $B_{k}=A / \pi^{k} A$ are Frobenius for $k \geqslant 1$. If $\mathcal{O} / \pi \mathcal{O}$ is a finite ring, then all $B_{k}$ are finite Frobenius rings.

Theorem 2.3 and Corollary 2.1 indicate the importance of the study of Gorenstein matrices.

2.3. Quivers. Following P.Gabriel a quiver is a finite directed graph.

A quiver $Q=(V Q, A Q, s, e)$ is a finite directed graph which consists of finite sets $V Q$ and $A Q$ and two mappings $s, e: A Q \rightarrow V Q$. The elements of $V Q$ are called vertices (or points), and those of $A Q$ are called arrows. 
Usually, the set of vertices $V Q$ will be a set $\{1,2, \ldots, n\}$. We say that each arrow $\sigma \in A Q$ starts at the vertex $s(\sigma)$ and ends at the vertex $e(\sigma)$. The vertex $s(\sigma)$ is called the start (or initial, or source) vertex and the vertex $e(\sigma)$ is called the end (or target) vertex of $\sigma$.

A quiver without multiple arrows and multiple loops is called a simply laced quiver.

Assume that we have $t_{i j}$ arrows from the vertex $i$ to the vertex $j$. The $(n \times n)$-matrix $[Q]=\left(t_{i j}\right)$ is called the adjacency matrix of the quiver $Q$. A quiver $Q$ is simply laced if and only if its adjacency matrix $[Q]$ is $(0,1)$-matrix.

Let $e_{i j}, i, j=1, \ldots, n$, be the matrix units in $M_{n}(\mathbb{R})$, where $\mathbb{R}$ is the field of real numbers, $B=\sum_{i, j=1}^{n} b_{i j} e_{i j} \in M_{n}(\mathbb{R})$.

Recall that the quiver $Q=Q(B)$ of a matrix $B=\left(b_{i j}\right)$ is the simply laced quiver with $V Q=\{1, \ldots, n\}$ and there exists the arrow $\sigma: i \rightarrow j$ if and only if $b_{i j} \neq 0$.

Let $\tau: i \rightarrow \tau(i)$ is a permutation of $\{1, \ldots, n\}$. A matrix $P_{\tau}=\sum_{i=1}^{n} e_{i \tau(i)}$ is called a permutation matrix corresponding to $\tau$.

Two quivers $Q_{1}$ and $Q_{2}$ are called isomorphic $\left(Q_{1} \simeq Q_{2}\right)$ if there is a bijective correspondence between vertices and arrows such that starts and ends of corresponding arrows map into each other. In this case there exists a permutation matrix $P_{\tau}$ such that $\left[Q_{2}\right]=P_{\tau}^{T}\left[Q_{1}\right] P_{\tau}$, where $T$ denotes the transpose.

Conversely, if $\left[Q_{2}\right]=P_{\tau}^{T}\left[Q_{1}\right] P_{\tau}$, then $Q_{1} \simeq Q_{2}$.

Let $[Q]$ be the adjacency matrix of a quiver $Q$ and $[Q] \in M_{n}(\mathbb{C})$, where $\mathbb{C}$ is the field of the complex numbers.

Let $\vec{z}^{T}=\left(z_{1}, \ldots, z_{n}\right)^{T} \in \mathbb{C}^{n}$ be a right eigenvector of $[Q]$ with an eigenvalue $\lambda$, i.e.,

$$
[Q] \vec{z}^{T}=\lambda \vec{z}^{T}
$$

and $\vec{u}=\left(u_{1}, \ldots, u_{n}\right) \in \mathbb{C}^{n}$ be a left eigenvector of $[Q]$ with an eigenvalue $\mu$, i.e.,

$$
\vec{u}[Q]=\lambda \vec{u} .
$$

Let $\tau$ be a permutation of the set $\{1, \ldots, n\}$ and $\vec{a}=\left(a_{1}, \ldots, a_{n}\right) \in \mathbb{C}^{n}$.

Denote by $\vec{a}_{\tau}$ the $n$-dimensional vector which is obtained from $\vec{a}=$ $\left(a_{1}, \ldots, a_{n}\right)$ by the permutation of its coordinates by the rule $\vec{a}_{\tau}=\left(a_{\tau(1)}, \ldots\right.$, 
$\left.a_{\tau(n)}\right)$. Two $n$-dimensional vectors $\vec{a}$ and $\vec{b}$ are called equivalent if $\vec{b}=\vec{a}_{\tau}$ for some permutation $\tau$.

Proposition 2.1. Let $Q_{1}$ and $Q_{2}$ are two isomorphic quivers. Let $\vec{a}^{T}$ be the right eigenvector of the matrix $\left[Q_{2}\right]$ with the eigenvalue $\lambda$. Then the vector $\vec{a}_{\tau}^{T}$ is the right eigenvector of the matrix $\left[Q_{1}\right]$ with the same eigenvalue $\lambda$. If $\vec{b}^{T}$ is right eigenvector of $\left[Q_{1}\right]$ with eigenvalue $\lambda$ then $\vec{b}_{\tau^{-1}}^{T}$ is the right eigenvector of $\left[Q_{2}\right]$ with eigenvalue $\lambda$.

Proof. The equality $P_{\tau}\left[Q_{2}\right]=\left[Q_{1}\right] P_{\tau}$ holds. Let $\vec{a}^{T}$ be an eigenvector of the matrix $\left[Q_{2}\right]$ with eigenvalue $\lambda$. Then $P_{\tau}\left[Q_{2}\right] \vec{a}^{T}=\lambda P_{\tau} \vec{a}^{T}=\lambda \vec{a}_{\tau}^{T}=$ $\left[Q_{1}\right] P_{\tau} \vec{a}^{T}=\left[Q_{1}\right] \vec{a}_{\tau}^{T}$, i.e. $\vec{a}_{\tau}^{T}$ is eigenvector of the matrix $\left[Q_{1}\right]$.

Let $\vec{b}$ be an eigenvector of the matrix $\left[Q_{1}\right]$ with eigenvalue $\lambda$. From $\left[Q_{2}\right] P_{\tau}^{-1}=P_{\tau_{-1}}\left[Q_{1}\right]$ follows $\left[Q_{2}\right] P_{\tau^{-1}} \vec{b}^{T}=P_{\tau^{-1}}\left[Q_{1}\right] \vec{b}^{T}=\lambda P_{\tau^{-1}} \vec{b}^{T}=$ $\lambda \vec{b}_{\tau^{-1}}^{T}=\left[Q_{2}\right] b_{\tau^{-1}}^{T}$.

Definition 2.4. The characteristic polynomial $\chi_{Q}(x)$ of the quiver $Q$, is called the characteristic polynomial of the matrix $[Q]$, i.e., $\chi_{Q}(x)=$ $\operatorname{det}(x E-[Q])$.

Obviously, if $Q_{1} \simeq Q_{2}$, then $\chi_{Q_{1}}(x)=\chi_{Q_{2}}(x)$.

Recall that path from the vertex $i$ to the vertex $j$ of the quiver $Q$ is called the a sequence of arrows $\sigma_{1} \ldots \sigma_{r}$ such that the start vertex of each arrow $\sigma_{m}$ coincides with the end vertex of the previous one $\sigma_{m-1}$ for all $m, 1<m \leqslant r$ and moreover, the vertex $i$ is the start vertex of $\sigma_{1}$, while the vertex $j$ if the end vertex $j$ is the end vertex of $\sigma_{r}$. The number $r$ of arrows is called the length of the path.

Definition 2.5. Let $Q$ be a quiver and $V Q=\{1, \ldots, n\}$. If $n \geqslant 2, Q$ is called strongly connected if for any two vertices there exists a path from one to another.

By convention a one-point quiver will be considered a strongly connected quiver.

\section{Perron and Frobenius theorems.}

In this section we recall classical theorems of Perron and Frobenius. Recall that a matrix $B \in M_{n}(\mathbb{R})$ is called permutationally reducible if there exists a permutation matrix $P_{\tau}$ such that

$$
P_{\tau}^{T} B P_{\tau}=\left(\begin{array}{cc}
B_{1} & B_{12} \\
0 & B_{2}
\end{array}\right)
$$


where $B_{1}$ and $B_{2}$ are square matrices of order less that $n$. Otherwise, the matrix $B$ is called permutationally irreducible.

From the equality

$$
D_{n}\left(\begin{array}{cc}
B_{1} & B_{12} \\
0 & B_{2}
\end{array}\right) D_{n}=\left(\begin{array}{cc}
B_{1}^{(1)} & 0 \\
B_{21} & B_{2}^{(2)}
\end{array}\right)
$$

it follows that $B$ is permutationally reducible if and only if there exists a permutation matrix $P_{\nu}$ such that

$$
P_{\nu}^{T} B P_{\nu}=\left(\begin{array}{cc}
B_{1}^{(1)} & 0 \\
B_{21} & B_{2}^{(2)}
\end{array}\right), \text { where } D_{n}=\sum_{i=1}^{n} e_{i, n-i+1}
$$

and $B_{1}^{(1)}$ and $B_{2}^{(2)}$ are square matrices of order less that $n$.

Proposition 3.1. $[4, \S 11.3]$ A matrix $B$ is permutationally irreducible if and only if the simply laced quiver $Q(B)$ is strongly connected.

A vector $\vec{y}=\left(y_{1}, \ldots, y_{n}\right) \in \mathbb{R}^{n}$ is called positive if $y_{i}>0$ for $i=$ $1, \ldots, n$. The number $\|\vec{y}\|=\sqrt{y_{1}^{2}+\ldots+y_{n}^{2}}$ is called the norm of vector $\vec{y}$.

We have the following well-known result.

Theorem 3.1 (Perron theorem). [6] A positive matrix $A=\left(a_{i j}\right)(i, j=$ $1, \ldots, n)$ always has a real and positive eigenvalue $r$ which is a simple root of the characteristic equation and which is larger that the absolute values of all other eigenvalues. To this maximal eigenvalue $r$ there corresponds a positive eigenvector $z=\left(z_{1}, z_{2}, \ldots, z_{n}\right)$ of $A$.

A positive matrix is a special case of a permutationally irreducible nonnegative matrix. Frobenius generalized the Perron theorem by investigating the spectral properties of permutationally irreducible non-negative matrices.

Theorem 3.2 (Frobenius theorem). [2] A permutationally irreducible nonnegative matrix $A=\left(a_{i j}\right) i, j=1, \ldots, n$ always has a positive eigenvalue $r$ which is a simple root of the characteristic equation. The absolute values of all the other eigenvalues do not exceed $r$. To the maximal eigenvalue $r$ there corresponds a positive eigenvector.

Moreover, if $A$ has $h$ eigenvalues $\lambda_{0}=r, \lambda_{1}, \ldots, \lambda_{h-1}$ of absolute value $r$, then these numbers are all distinct and are roots of the equation

$$
\lambda^{h}-r^{h}=0
$$


More generally: The whole spectrum $\lambda_{0}, \lambda_{1}, \ldots, \lambda_{h-1}$ of $A$, regarded as a system of points in the complex $\lambda$-plane, goes over into itself under a rotation of the plane by the angle $2 \pi / h$. If $h>1$, then, by means of a permutation, A can be brought into the following block cyclic form:

$$
A=\left(\begin{array}{cccccc}
0 & A_{12} & 0 & \ldots & \ldots & 0 \\
0 & \ddots & A_{23} & \ddots & & \vdots \\
\vdots & \ddots & \ddots & \ddots & \ddots & \vdots \\
\vdots & & \ddots & \ddots & \ddots & 0 \\
0 & \ldots & \ldots & 0 & \ddots & A_{h-1, h} \\
A_{h 1} & 0 & \ldots & \ldots & 0 & 0
\end{array}\right)
$$

where there are square blocks along the main diagonal.

A strongly connected quiver $Q$ is called primitive if its adjacency matrix $[Q]$ has only one eigenvalue with maximal absolute value $r$, otherwise $Q$ is called imprimitive.

\section{Gorenstein quivers}

In this main section we discuss the properties of Gorenstein quivers.

We will need the following definition.

Definition 4.1. Let $Q$ be a strongly connected quiver with the adjacency matrix $[Q]$. The maximal positive eigenvalue $r$ is called the index of $Q$ $(r=i n x Q)$.

Remark 4.1. Let

$$
s_{i}=\sum_{j=1}^{n} a_{i j} \quad(i=1,2, \ldots, n), s=\min _{1 \leqslant i \leqslant n} s_{i}, S=\max _{1 \leqslant i \leqslant n} s_{i} .
$$

Then for a permutationally irreducible matrix $A \geqslant 0$

$$
s \leqslant r \leqslant S,
$$

and the equality sign on the left or the right of $r$ holds for $s=S$ only; i.e., they hold only when all the row-sums $s_{1}, s_{2}, \ldots, s_{n}$ are all equal. 
Remark 4.2. A permutationally irreducible matrix $A \geqslant 0$ cannot have two linearly independent positive eigenvectors with the maximal real eigenvalue $r$.

Let $\mathcal{E}=\left(\alpha_{i j}\right)$ be a reduced exponent matrix. Set $\mathcal{E}^{(1)}=\left(\beta_{i j}\right)$, where $\beta_{i j}=\alpha_{i j}$ for $i \neq j$ and $\beta_{i i}=1$ for $i=1 \ldots, n$. Also set $\mathcal{E}^{(2)}=\left(\gamma_{i j}\right)$, where $\gamma_{i j}=\min _{1 \leqslant k \leqslant n}\left(\beta_{i k}+\beta_{k i}\right)$. Obviously, $[Q]=\mathcal{E}^{(2)}-\mathcal{E}^{(1)}$ is a $(0,1)$-matrix.

Theorem 4.1. [4, Ch.14] The matrix $[Q]=\mathcal{E}^{(2)}-\mathcal{E}^{(1)}$ is the adjacency matrix of the strongly connected simply laced quiver $Q=Q(\mathcal{E})$.

Definition 4.2. A quiver $Q$ is called Gorenstein if it is the quiver of a Gorenstein matrix.

We immediately obtain from Theorem 4.1 the following property of Gorenstein quivers.

Corollary 4.1. A Gorenstein quiver $Q$ is strongly connected simply laced quiver.

Eigenvector of a matrix $[Q]$ is also called an eigenvector of the quiver $Q$.

Corollary 4.2. A Gorenstein quiver $Q$ has a positive eigenvector.

Example 4.1. Consider the matrix

$$
\mathcal{E}_{12}=\left(\begin{array}{cccccccccccc}
0 & 6 & 4 & 4 & 4 & 4 & 3 & 3 & 2 & 2 & 3 & 3 \\
6 & 0 & 4 & 4 & 4 & 4 & 3 & 3 & 2 & 2 & 3 & 3 \\
2 & 2 & 0 & 6 & 4 & 4 & 2 & 2 & 4 & 4 & 2 & 2 \\
2 & 2 & 6 & 0 & 4 & 4 & 2 & 2 & 4 & 4 & 2 & 2 \\
2 & 2 & 2 & 2 & 0 & 6 & 4 & 4 & 4 & 4 & 2 & 2 \\
2 & 2 & 2 & 2 & 6 & 0 & 4 & 4 & 4 & 4 & 2 & 2 \\
3 & 3 & 4 & 4 & 2 & 2 & 0 & 6 & 4 & 4 & 4 & 4 \\
3 & 3 & 4 & 4 & 2 & 2 & 6 & 0 & 4 & 4 & 4 & 4 \\
4 & 4 & 2 & 2 & 2 & 2 & 2 & 2 & 0 & 6 & 4 & 4 \\
4 & 4 & 2 & 2 & 2 & 2 & 2 & 2 & 6 & 0 & 4 & 4 \\
3 & 3 & 4 & 4 & 4 & 4 & 2 & 2 & 2 & 2 & 0 & 6 \\
3 & 3 & 4 & 4 & 4 & 4 & 2 & 2 & 2 & 2 & 6 & 0
\end{array}\right)
$$

This is the Gorenstein matrix with the following permutation

$$
\sigma\left(\mathcal{E}_{12}\right)=(1,2)(3,4)(5,6)(7,8)(9,10)(11,12) .
$$

Now we construct the Gorenstein quiver $Q(\mathcal{E})$ of $\mathcal{E}_{12}$.

$\mathcal{E}_{12}^{(1)}=E_{12}+\mathcal{E}_{12}$, where $E_{12}$ is the identity $12 \times 12$-matrix, 


$$
\mathcal{E}_{12}^{(2)}=\left(\begin{array}{cccccccccccc}
2 & 6 & 4 & 4 & 4 & 4 & 4 & 4 & 3 & 3 & 4 & 4 \\
6 & 2 & 4 & 4 & 4 & 4 & 4 & 4 & 3 & 3 & 4 & 4 \\
3 & 3 & 2 & 6 & 4 & 4 & 3 & 3 & 4 & 4 & 3 & 3 \\
3 & 3 & 6 & 2 & 4 & 4 & 3 & 3 & 4 & 4 & 3 & 3 \\
3 & 3 & 3 & 3 & 2 & 6 & 4 & 4 & 4 & 4 & 3 & 3 \\
3 & 3 & 3 & 3 & 6 & 2 & 4 & 4 & 4 & 4 & 3 & 3 \\
4 & 4 & 4 & 4 & 3 & 3 & 2 & 6 & 5 & 5 & 4 & 4 \\
4 & 4 & 4 & 4 & 3 & 3 & 6 & 2 & 5 & 5 & 4 & 4 \\
4 & 4 & 3 & 3 & 3 & 3 & 3 & 3 & 2 & 6 & 4 & 4 \\
4 & 4 & 3 & 3 & 3 & 3 & 3 & 3 & 6 & 2 & 4 & 4 \\
4 & 4 & 4 & 4 & 4 & 4 & 3 & 3 & 3 & 3 & 2 & 6 \\
4 & 4 & 4 & 4 & 4 & 4 & 3 & 3 & 3 & 3 & 6 & 2
\end{array}\right)
$$

and

$$
\left[Q\left(\mathcal{E}_{12}\right)\right]=\left(\begin{array}{cccccccccccc}
1 & 0 & 0 & 0 & 0 & 0 & 1 & 1 & 1 & 1 & 1 & 1 \\
0 & 1 & 0 & 0 & 0 & 0 & 1 & 1 & 1 & 1 & 1 & 1 \\
1 & 1 & 1 & 0 & 0 & 0 & 1 & 1 & 0 & 0 & 1 & 1 \\
1 & 1 & 0 & 1 & 0 & 0 & 1 & 1 & 0 & 0 & 1 & 1 \\
1 & 1 & 1 & 1 & 1 & 0 & 0 & 0 & 0 & 0 & 1 & 1 \\
1 & 1 & 1 & 1 & 0 & 1 & 0 & 0 & 0 & 0 & 1 & 1 \\
1 & 1 & 0 & 0 & 1 & 1 & 1 & 0 & 1 & 1 & 0 & 0 \\
1 & 1 & 0 & 0 & 1 & 1 & 0 & 1 & 1 & 1 & 0 & 0 \\
0 & 0 & 1 & 1 & 1 & 1 & 1 & 1 & 1 & 0 & 0 & 0 \\
0 & 0 & 1 & 1 & 1 & 1 & 1 & 1 & 0 & 1 & 0 & 0 \\
1 & 1 & 0 & 0 & 0 & 0 & 1 & 1 & 1 & 1 & 1 & 0 \\
1 & 1 & 0 & 0 & 0 & 0 & 1 & 1 & 1 & 1 & 0 & 1
\end{array}\right)
$$

Obviously, inx $\mathcal{E}_{12}=7$, but the sum of all elements of the first column is 9 .

The right eigenvector of $[Q(\mathcal{E})]$ is $(1,1,1,1,1,1,1,1,1,1,1,1)^{T}$.

Our computations show that left eigenvector of $\left[Q\left(\mathcal{E}_{12}\right)\right]$ which corresponds to the eigenvalue 7 is

$$
(15,15,8,8,10,10,16,16,14,14,11,11) .
$$

We have the following statement.

Proposition 4.1. Let $Q$ be a simply laced strongly connected quiver, $d$ be an arbitrary positive real number. There exists a unique right positive eigenvector $\vec{u}$ with the eigenvalue $r=\operatorname{inx} Q$ and $\|\vec{u}\|=d$.

Proof. By the Frobenius theorem there exists a positive right eigenvector $\vec{x}$ with the eigenvalue $r$. Let $\|\vec{x}\|=d_{1}$, then $\left\|d d_{1}^{-1} \vec{x}\right\|=d$. Let $\vec{u}$ and $\vec{v}$ be two positive eigenvectors with the eigenvalue $r$, and $\|\vec{u}\|=\|\vec{v}\|=d$. By 
Proposition 3.1 and Remark $4.2 \vec{u}=\alpha \vec{v}$. We have $\|\vec{u}\|=\alpha\|\vec{v}\|=\alpha\|\vec{u}\|$. Therefore, $\alpha=1$ and $\vec{u}=\vec{v}$.

Now we can establish our main result.

Theorem 4.2. Let $Q_{1}$ and $Q_{2}$ be two isomorphic simply laced strongly connected quivers. Then their characteristic polynomials $\chi_{Q_{1}}(x)$ and $\chi_{Q_{2}}(x)$ are equal and positive right (left) eigenvectors $\vec{a}$ and $\vec{b}$ with the maximal eigenvalue $r$ such that $\|\vec{a}\|=\|\vec{b}\|$ are equivalent.

Proof. We have that $\left[Q_{2}\right]=P_{\tau}^{T}\left[Q_{1}\right] P_{\tau}$. Therefore, $\chi_{Q_{1}}(x)=\chi_{Q_{2}}(x)$. Let $\vec{a}$ be a right eigenvector of $\left[Q_{2}\right]$ with the eigenvalue $r$, and $\vec{b}$ be a right eigenvector of $\left[Q_{2}\right]$ with the eigenvalue $r$. By Proposition 2.1, $\vec{b}$ is the right eigenvector of $\left[Q_{1}\right]$ with the eigenvalue $r$. We have that $\left\|\vec{b}_{\tau}\right\|=\|\vec{b}\|$ for any $\tau$. Therefore, $\left\|\vec{b}_{\tau}\right\|=\|\vec{a}\|$. Applying Proposition 4.1 we obtain $\vec{a}=\vec{b}_{\tau}$.

Let $Q$ be a simply laced strongly connected quiver with the adjacency matrix $[Q]=\left(t_{i j}\right)$. The transpose quiver $Q^{T}$ is the quiver whose adjacency matrix $\left[Q^{T}\right]$ is equal $[Q]^{T}$. The quiver $Q^{T}$ is simply laced and strongly connected if and only if the quiver $Q$ has the same properties. Obviously, $Q_{1} \simeq Q_{2}$ if and only if $Q_{1}^{T}=Q_{2}^{T}$. If $\vec{b}$ is a left eigenvector of $[Q]^{T}$, then $\vec{b}^{T}$ is a right eigenvector of $[Q]$. So, the theorem is proved in the left case. Te right case is proven analogously.

Applying Theorem 4.2 to the case of Gorenstein quivers we obtain

Corollary 4.3. Let $Q_{1}$ and $Q_{2}$ be two Gorenstein quivers. If $Q_{1} \simeq Q_{2}$, then $\chi_{Q_{1}}(x)=\chi_{Q_{2}}(x), r=\operatorname{inx} Q_{1}=\operatorname{inx} Q_{2}$ and right (left) positive eigenvector $\vec{a}$ of $\left[Q_{1}\right]$ with the eigenvalue $r$ and right (left) positive eigenvector $\vec{b}$ of $\left[Q_{2}\right]$ with the same eigenvalue such that $\|\vec{a}\|=\|\vec{b}\|$ are equivalent.

Example 4.2. Consider two Gorenstein quivers: 
$Q_{1}$ :
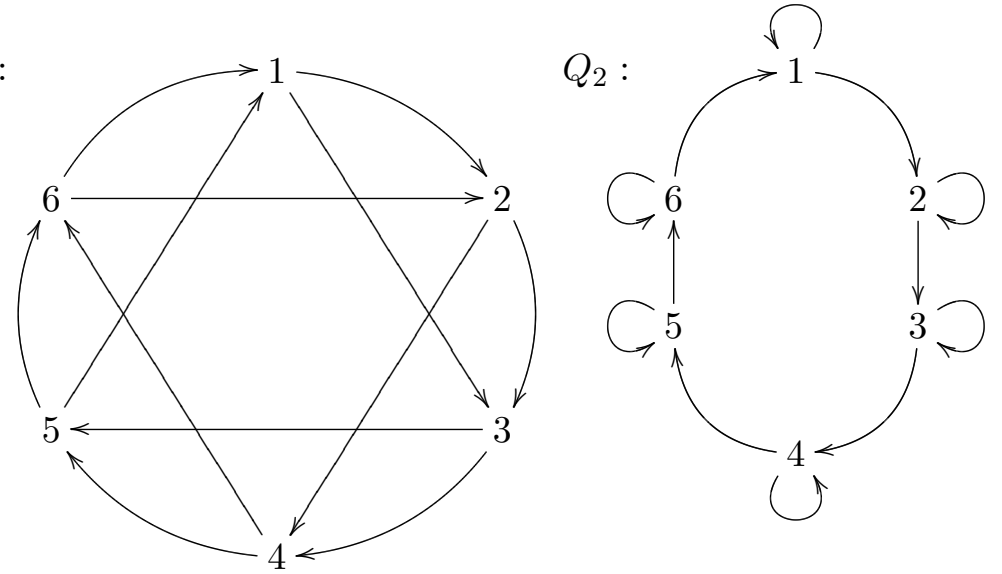

$Q_{1}=Q\left(\mathcal{E}_{6}\right)$, where

$$
\mathcal{E}_{6}=\left(\begin{array}{cccccc}
0 & 0 & 0 & 0 & 0 & 0 \\
2 & 0 & 1 & 0 & 1 & 0 \\
1 & 1 & 0 & 0 & 0 & 0 \\
2 & 1 & 2 & 0 & 1 & 0 \\
1 & 1 & 1 & 1 & 0 & 0 \\
2 & 1 & 2 & 1 & 2 & 0
\end{array}\right)
$$

is the Gorenstein matrix with the permutation

$$
\left(\begin{array}{llllll}
1 & 2 & 3 & 4 & 5 & 6 \\
6 & 1 & 2 & 3 & 4 & 5
\end{array}\right) .
$$

$Q_{2}=Q\left(T_{6}\right)$, where

$$
T_{6}=\left(\begin{array}{cccccc}
0 & 0 & 0 & 0 & 0 & 0 \\
2 & 0 & 0 & 0 & 0 & 0 \\
2 & 2 & 0 & 0 & 0 & 0 \\
2 & 2 & 2 & 0 & 0 & 0 \\
2 & 2 & 2 & 2 & 0 & 0 \\
2 & 2 & 2 & 2 & 2 & 0
\end{array}\right)
$$

is the Gorenstein matrix with the same permutation

$$
\left(\begin{array}{llllll}
1 & 2 & 3 & 4 & 5 & 6 \\
6 & 1 & 2 & 3 & 4 & 5
\end{array}\right) .
$$


Obviously, the adjacency matrices of these quivers are

$$
\left[Q_{1}\right]=\left(\begin{array}{cccccc}
0 & 1 & 1 & 0 & 0 & 0 \\
0 & 0 & 1 & 1 & 0 & 0 \\
0 & 0 & 0 & 1 & 1 & 0 \\
0 & 0 & 0 & 0 & 1 & 1 \\
1 & 0 & 0 & 0 & 0 & 1 \\
1 & 1 & 0 & 0 & 0 & 0
\end{array}\right) \quad \text { and } \quad\left[Q_{2}\right]=\left(\begin{array}{cccccc}
1 & 1 & 0 & 0 & 0 & 0 \\
0 & 1 & 1 & 0 & 0 & 0 \\
0 & 0 & 1 & 1 & 0 & 0 \\
0 & 0 & 0 & 1 & 1 & 0 \\
0 & 0 & 0 & 0 & 1 & 1 \\
1 & 0 & 0 & 0 & 0 & 1
\end{array}\right)
$$

We have that inx $Q_{1}=$ inx $Q_{2}=2$. The left eigenvector $(1,1,1,1,1,1)$ of $\left[Q_{1}\right]$ is the left eigenvector of $\left[Q_{2}\right]$. The right eigenvector $(1,1,1,1,1,1)^{T}$ of $\left[Q_{1}\right]$ is the right eigenvector of $\left[Q_{2}\right]$. It is easy to see that $\chi_{Q_{1}}(x)=$ $(x+1)^{2} x(x-2)\left(x^{2}+3\right)$ and $\chi_{Q_{2}}(x)=x(x-2)\left(x^{4}-4 x^{3}+\lambda x^{2}-6 x+3\right)$. By Theorem 4.2 the quivers $Q_{1}$ and $Q_{2}$ are non-isomorphic.

\section{References}

[1] Bass, H. On the ubiquity of Gorenstein rings, Math. Zeit., V.82, 1963, p.8-28

[2] Frobenius, G. Uber Matrizen aus nicht negativen Elementen, S.-B. Deutsch Akad. Wiss. Berlin. Mat-Nat. Kl., 1912, p.456-477

[3] Gantmakher, F.R. Applications of theory of matrices, Interscience Publishers, New York, 1959.

[4] Hazewinkel, M.; Gubareni, N.; Kirichenko, V. V. Algebras, rings and modules. Vol. 1. Mathematics and Its Applications 575, Kluwer Academic Publisher, 2004. xii+380 p.

[5] Hazewinkel, M.; Gubareni, N.; Kirichenko, V. V. Algebras, rings and modules. Vol. 2. Mathematics and Its Applications (Springer), 586. Springer, Dordrecht, 2007. xii+400 p.

[6] Perron O. Uber Matrizen, Math. Ann., v.64, 1907, p. 248-263 